\title{
Impact of exercise training on oxidative stress in individuals with a spinal cord injury
}

\author{
Noortje van Duijnhoven · Evelyne Hesse • \\ Thomas Janssen • Will Wodzig • Peter Scheffer • \\ Maria Hopman
}

Accepted: 8 February 2010/Published online: 3 April 2010

(c) The Author(s) 2010. This article is published with open access at Springerlink.com

\begin{abstract}
Individuals with a spinal cord injury (SCI) have an increased cardiovascular risk. We hypothesize that (anti)oxidative imbalance is associated with the increased cardiovascular risk in SCI, while exercise can reverse this status. The aim of the study is to compare baseline levels of oxidative stress and antioxidative capacity between individuals with $\mathrm{SCI}$ and able-bodied (AB) subjects, and to assess acute and long-term effects of functional electrical stimulation (FES) exercise on oxidative stress and antioxidative capacity in SCI. Venous blood was taken from subjects with an SCI $(n=9)$ and age- and gender-matched $\mathrm{AB}$ subjects $(n=9)$ to examine oxidative stress through
\end{abstract}

Communicated by William Kraemer.

N. van Duijnhoven · E. Hesse · M. Hopman ( $\square)$

Department of Physiology, Radboud University Nijmegen

Medical Centre, Geert Grooteplein-Noord 21,

6525 EZ Nijmegen, The Netherlands

e-mail: M.Hopman@fysiol.umcn.nl

E. Hesse

Institute of Human Movement Sciences and Sport, ETH Zurich,

Zurich, Switzerland

T. Janssen

Faculty of Human Movement Sciences, Research Institute

MOVE, VU University, Amsterdam, The Netherlands

T. Janssen

Rehabilitation Centre, Amsterdam, The Netherlands

W. Wodzig

Department of Clinical Chemistry, Maastricht University

Medical Centre, Maastricht, The Netherlands

P. Scheffer

Department of Clinical Chemistry, VU University Medical

Centre, Amsterdam, The Netherlands malondialdehyde (MDA) levels, while superoxide dismutase (SOD) and glutathione peroxidase (GPx) enzyme levels represented anti-oxidative capacity. Subsequently, subjects with an SCI performed an 8-week FES exercise training period. Blood was taken before and after the first exercise bout and after the last FES session to examine the acute and chronic effect of FES exercise, respectively. Baseline levels of MDA, SOD and GPx were not different between individuals with $\mathrm{SCI}$ and $\mathrm{AB}$ subjects. SCI demonstrated a correlation between initial fitness level and MDA ( $R=-0.83, P=0.05$ ). MDA, SOD and GPx levels were neither altered by a single FES exercise bout nor by 8 weeks FES training. In conclusion, although individuals with an SCI demonstrate a preserved (anti)oxidative status, the correlation between fitness level and (anti)oxidative balance suggests that higher fitness levels are related to improved (anti)oxidative status in SCI. Nonetheless, the FES exercise stimulus was insufficient to acutely or chronically change (anti)oxidative status in individuals with an SCI.

Keywords Oxidative stress - Antioxidative capacity · Functional electrical stimulation $\cdot$ Spinal cord injury

\section{Introduction}

The prevalence of morbidity and mortality for cardiovascular diseases (CVDs) in individuals with a chronic spinal cord injury (SCI) is high relative to ambulatory subjects (Myers et al. 2007). Physical inactivity, which is an independent risk factor of CVD and a central characteristic in individuals having an SCI below the lesion, is likely to accelerate the atherosclerotic process and the consequent increase in cardiovascular risk (Laufs et al. 2005). 
Interestingly, the increased cardiovascular risk in SCI is unlikely explained by traditional cardiovascular risk factors (i.e. hyperlipidemia and hyperglycemia) (Liang et al. 2007).

A more novel factor is oxidative stress and/or decreased antioxidative capacity in the vascular wall (Yorek 2003). Possibly, an imbalance in (anti)oxidative status may contribute to the increased prevalence of cardiovascular diseases in SCI. The idea of an increased oxidative stress in SCI is further based on the recently described relation between fitness level and oxidative stress in able-bodied subjects (Bloomer and Fisher-Wellman 2008). However, previous studies that examined oxidative stress in individuals with an SCI presented conflicting results, included no other cardiovascular risk factors or lacked the inclusion of able-bodied controls (Kocak et al. 2005; Wozniak et al. 2003). Therefore, the first aim of the present study was to compare baseline levels of oxidative stress and antioxidative capacity from individuals with a chronic SCI with levels in able-bodied subjects. We hypothesized that individuals with an SCI have an increased oxidative stress and a decreased antioxidative capacity compared to able-bodied individuals.

Physical exercise in able-bodied subjects immediately increases levels of oxidative stress (Ji 1999; Powers and Jackson 2008), and subsequently leads to elevated antioxidative enzyme activity (Alessio 1993; Laughlin et al. 1990; Powers and Jackson 2008). Muscle activation by functional electrical stimulation (FES) in individuals with an SCI is demonstrated to immediately increase oxygen consumption and heart rate (Holme et al. 2001), and also represents an efficient manner to alter vascular parameters in the paralyzed limbs in SCI, such as arterial diameter and compliance, blood flow, endothelial function and vascular resistance (de Groot et al. 2005; Hopman et al. 2002; Thijssen et al. 2006). Accordingly, we expected FES exercise to immediately alter oxidative stress, while continuing FES cycling training will improve (anti)oxidative status in SCI. Therefore, the second aim of this study was to assess the acute and long-term effects of electrically induced exercise on oxidative stress and antioxidative capacity in individuals with a chronic SCI.

\section{Methods}

\section{Subjects}

Nine men with an SCI (41 \pm 9 years) and nine age- and gender-matched, able-bodied (AB) subjects ( $42 \pm 10$ years) participated in this study (Table 1). Seven subjects with SCI had a complete lesion, varying between C5 and T11 [ASIA Impairment Scale (AIS) grade A], while two subjects had an incomplete lesion at C5 (AIS grades B and D). All lesions were traumatic and existed for at least 4 years. None of the participants had any cardiovascular disease, diabetes (fasting glucose $>6.0 \mathrm{mmol} / \mathrm{l}$ ) nor cardiovascular risk factors such as hypercholesterolemia $(>6.5 \mathrm{mmol} / \mathrm{l})$ and hypertension. Before entering the study, subjects with SCI were physically screened for cardiac and pulmonary abnormalities and cardiovascular disease. None of the subjects received medication or vitamin supplementation known to interfere with the (anti)oxidative or cardiovascular system. The study was approved by the ethics committee of the Radboud University Nijmegen Medical Centre, and conforms with the principles outlined in the Declaration of Helsinki. All subjects provided written informed consent prior to testing.

\section{Procedures}

First, baseline blood was taken for a cross-sectional comparison on oxidative stress and antioxidative capacity between individuals with $\mathrm{SCI}$ and $\mathrm{AB}$ subjects. Second, an intervention study was performed on the participants with SCI, which consisted of an 8-week FES exercise training period. Before and after the first FES cycling bout and after the 8-week intervention, blood samples were taken in subjects with SCI to assess the acute and chronic effect of FES cycling on oxidative stress and antioxidative capacity.

\section{Measurements}

Oxidative stress was indirectly estimated by the moststudied end-product of lipid peroxidation of polyunsaturated fatty acids, malondialdehyde (MDA), from venous blood. Antioxidative capacity is represented by the activity of superoxide dismutase (SOD) and glutathione peroxidase (GPx), two main antioxidative enzymes in the human body. All blood samples were taken at least $48 \mathrm{~h}$ post-exercise

Table 1 Subject characteristics

\begin{tabular}{lll}
\hline & SCI $(n=9)$ & AB $(n=9)$ \\
\hline Age (years) & $41 \pm 9$ & $42 \pm 10$ \\
Body mass (kg) & $74 \pm 13$ & $79 \pm 18$ \\
Body height (cm) & $181 \pm 5$ & $180 \pm 8$ \\
Body mass index (kg/m $\left.{ }^{2}\right)$ & $22.8 \pm 3.2$ & $24.1 \pm 3.7$ \\
Resting heart rate (bpm) & $60 \pm 7$ & $60 \pm 9$ \\
Systolic blood pressure (mmHg) & $117 \pm 15$ & $128 \pm 15$ \\
Diastolic blood pressure (mmHg) & $75 \pm 9$ & $74 \pm 9$ \\
Sport activities (h/week) & $1.5[0-6]$ & $2.0[0-5]$ \\
\hline
\end{tabular}

Data are presented as mean $\pm \mathrm{SD}$, sport activities are presented as median [range]

$S C I$ spinal cord injury, $A B$ able bodied 
(except the post-exercise blood sample to assess the acute effects of FES cycling, which was taken immediately after cessation of the session), after at least $4 \mathrm{~h}$ fast and $12 \mathrm{~h}$ abstinence from caffeine and alcohol.

Physical fitness was assessed before and after the 8-week FES training period by an incremental maximal exercise test on an arm ergometer (Lode, Angio300, Groningen, The Netherlands) using a multistage protocol (workload increased by $10 \mathrm{~W} / \mathrm{min}$, starting at $10 \mathrm{~W}$, until exhaustion).

\section{Exercise intervention}

For the FES exercise training, a computer-controlled leg cycle ergometer (Ergys 2, Therapeutic Alliances Inc., USA) was used. Six self-adhesive $50 \times 90-\mathrm{mm}$ surface electrodes (Stimex, Pierenkemper GmbH, Germany) were placed on both legs over the quadriceps, hamstrings, and gluteal muscles. Electrical stimulation (duration monophasic square wave pulses, $450 \mu$ s, frequency $30 \mathrm{~Hz}$ ) was applied in coordinated sequence permitting cyclic patterns of muscle contractions resulting in leg cycling. The device was programmed to gradually increase the stimulation current amplitude, to a maximum of $140 \mathrm{~mA}$, to achieve a target pedaling rate of $\sim 50 \mathrm{rpm}$. The pedal resistance of the ergometer could be altered with $1 / 8 \mathrm{kp}$ increments (corresponding with $\sim 6.1 \mathrm{~W}$ at $50 \mathrm{rpm}$ ). Resistance was automatically reduced when pedaling rate dropped below $45 \mathrm{rpm}$, and the stimulation stopped when pedaling rate dropped below $35 \mathrm{rpm}$.

The FES exercise training started at a frequency of two times per week during the first 4 weeks, which was increased to three times per week during the last 4 weeks, leading to a total of 20 cycling training sessions per person. Muscles were stimulated for $30 \mathrm{~min}$ maximally. When subjects with SCI completed a session in $<30 \mathrm{~min}$, repeated bouts were performed until 30 min were achieved or a total of five bouts were completed.

\section{Data analysis}

After withdrawal, blood samples for determination of MDA, SOD and GPx levels were treated accordingly.

$M D A$ The level of MDA was determined after reaction with thiobarbituric acid (TBA) with an added alkaline hydrolysis step as described elsewhere (van de Kerkhof et al. 2004). In brief, blood was centrifuged at 3,700 rpm for $15 \mathrm{~min}$ at $4{ }^{\circ} \mathrm{C}$ and plasma was stored at $-80^{\circ} \mathrm{C}$ until further use. To $50 \mu \mathrm{l}$ of plasma $25 \mu \mathrm{l}$ of $0.2 \%$ butylated hydroxytoluene and $0.4 \mathrm{ml} 1 \mathrm{M}$ sodium hydroxide was added. The mixture was incubated at $60^{\circ} \mathrm{C}$ for $60 \mathrm{~min}$ in a shaking water bath. After cooling to room temperature,
$1.5 \mathrm{ml}$ of $10 \%$ trichloroacetic acid containing $1 \%$ potassium iodide was added, and the mixture was placed on ice for $10 \mathrm{~min}$ and centrifuged at $1,500 \mathrm{~g}$ for $10 \mathrm{~min}$ at $4^{\circ} \mathrm{C}$. To $0.5 \mathrm{ml}$ of the supernatant, $0.25 \mathrm{ml} 0.6 \%$ TBA was added, and the mixture was heated at $95^{\circ} \mathrm{C}$ for $30 \mathrm{~min}$. After cooling to room temperature and centrifugation $(1,500 \mathrm{~g}$, $10 \mathrm{~min}$ ), $50 \mu \mathrm{l}$ of the supernatant was injected into a symmetry C-18 column $(4.6 \times 100 \mathrm{~mm}, 3.5 \mu \mathrm{m}$; Waters 2695 Alliance Separations Module, Waters, USA) eluted at $1 \mathrm{ml} / \mathrm{min}$ using $70 \%(\mathrm{v} / \mathrm{v}) 25 \mathrm{mmol} / \mathrm{l}$ potassium dihydrogenphosphate ( $\mathrm{pH}$ 6.8) and $30 \%(\mathrm{v} / \mathrm{v})$ methanol. Detection of the MDA-TBA adduct was performed with fluorescence detection (excitation at $515 \mathrm{~nm}$ and emission at $553 \mathrm{~nm}$; Waters 474 Scanning Fluorescence Detector, Waters, USA). For quantification, intensities of the MDA-TBA peak areas were compared with a standard curve constructed with tetraethoxypropane (Sigma T9889).

SOD Blood $(0.5 \mathrm{ml})$ was centrifuged at $3,700 \mathrm{rpm}$ for $15 \mathrm{~min}$ at $4^{\circ} \mathrm{C}$. The separated plasma was aspirated and blood cells were washed with $3 \mathrm{ml}$ of $0.9 \%$ sodium chloride and centrifuged at $3,000 \mathrm{rpm}$ for $10 \mathrm{~min}$ at $4^{\circ} \mathrm{C}$. This step was repeated three times. $2.5 \mathrm{ml}$ cold Milli-Q water (Milli-Q Academic A10, Millipore, USA) was added after the washing steps and the sample was kept $15 \mathrm{~min}$ at $4^{\circ} \mathrm{C}$ before storage at $-80^{\circ} \mathrm{C}$. Before determination, the sample was diluted with $0.01 \mathrm{~mol} / \mathrm{l}$ phosphate buffer $\mathrm{pH} 7.0$ (Ransod, Randox, UK). The samples were detected at $500 \mathrm{~nm}$ and $37^{\circ} \mathrm{C}$ (Cobas Mira Plus, Roche Diagnostics Ltd., Switzerland). SOD activity was expressed as U/g Hb, where $\mathrm{Hb}$ was determined photometrically from $20 \mu \mathrm{l}$ of whole blood (HemoCue Plasma/Low Hb Photometer, HemoCue AB, Sweden).

GPx Whole blood samples were prepared manually (Ransel, Randox, UK) by diluting $0.05 \mathrm{ml}$ of the blood with $1 \mathrm{ml}$ diluting agent and incubated for $5 \mathrm{~min}$. Hemoglobin reagent (potassium phosphate $10.3 \mathrm{mmol} / \mathrm{l}$, potassium ferricyanide $6.08 \mathrm{mmol} / \mathrm{l}$, potassium cyanide $7.68 \mathrm{mmol} / \mathrm{l}$, surfactant $0.1 \% \mathrm{v} / \mathrm{v}$ ) was diluted with redistilled water (1:24), and $1 \mathrm{ml}$ was added to the diluted blood. GPx activity was measured by UV spectroscopy at $340 \mathrm{~nm}$ and $37^{\circ} \mathrm{C}$ (Cobas Mira Plus, Roche Diagnostics Ltd., Switzerland). GPx concentration was calculated from the following formula: U/l of hemolysate $=6,699 \times$ $\Delta A / \mathrm{min}$, where $\Delta A$ is the absorbance change per minute at $340 \mathrm{~nm}$. If $\Delta A$ exceeded 0.1 , the sample was diluted accordingly and detected again.

Statistical analysis

Statistical analyses were performed using SPSS 16.0 for Windows (SPSS, Chicago, USA). To assess differences 
between SCI and AB in subject characteristics and blood parameters, an unpaired Student's $t$ test was used. This test was not performed on BMI values, due to the recent finding that the BMI scale for people with chronic SCI does not correspond to the same absolute values of $\mathrm{AB}$ individuals (Laughton et al. 2009). In addition, we have examined the relation between fitness level (maximal workload corrected for body mass) and oxidative stress in SCI using a Pearson's correlation coefficient. The acute and long-term effects of FES exercise training on levels of MDA, SOD and GPx was tested with a paired Student's $t$ test. Statistical significance was set at $P \leq 0.05$. Values are presented as mean \pm standard deviation (SD), unless stated otherwise.

\section{Results}

\section{SCI versus $A B$}

Subject characteristics were not different between individuals with SCI and AB subjects (Table 1). Baseline levels of MDA, SOD and GPx were not different between individuals with $\mathrm{SCI}$ and $\mathrm{AB}$ subjects $(P=0.55,0.70$ and 0.73 , respectively; Fig. 1a-c). Individuals with SCI demonstrated a significant correlation between baseline levels of maximal workload achieved on the arm ergometer and MDA $(R=-0.83, P=0.05$; Fig. 2).

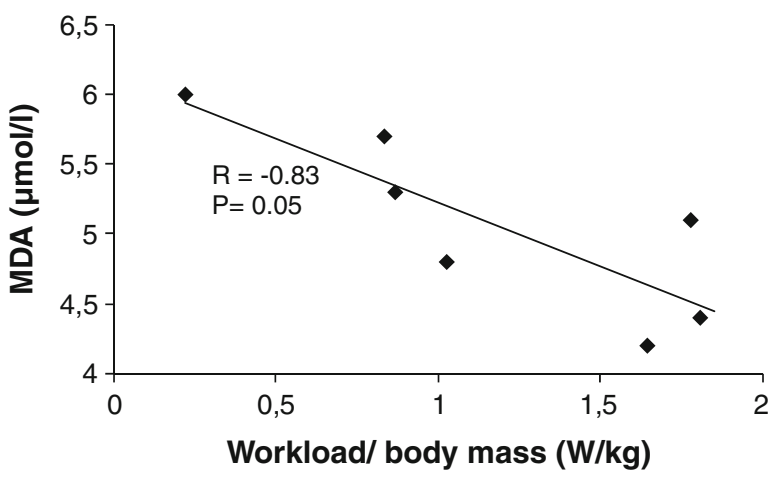

Fig. 2 Baseline levels of physical fitness [maximal workload arm ergometer corrected for body mass $(\mathrm{W} / \mathrm{kg})]$ and malondialdehyde (MDA) in individuals with spinal cord injury individuals $(n=7$; one subject with a cervical lesion was unable to perform the arm crank test; one subject showed MDA level $>3$ SD and was therefore defined as an outlier). $R$ derived from Pearson correlation

\section{Effect of FES cycling}

All individuals with SCI successfully completed the training period. Workload (median [25-75\%]) during FES exercise increased significantly from $0.0[0.0-2.2] \mathrm{kJ}$ to 11.0 [5.5-19.7] kJ after training (Wilcoxon: $P=0.008$ ). In addition, a strong trend for improvement was shown in maximal workload achieved during the arm ergometer test $(P=0.06)$.

A single, 30-min bout of FES exercise in individuals with SCI did not alter levels of MDA, SOD and GPx
Fig. 1 Resting levels of MDA malondialdehyde (a), SOD superoxide dismutase (b) and GPx gluthatione peroxidase (c) in able-bodied $(\mathrm{AB})$ subjects and individuals with a spinal cord injury (SCI) pre and post 8 weeks FES exercise training. Data are presented as individual blood values and mean \pm SEM. For $\mathrm{AB}, n=9$; for $\mathrm{SCI}, n=9$ in SOD, $n=8$ in MDA and GPx, due to one value identified as an outlier a

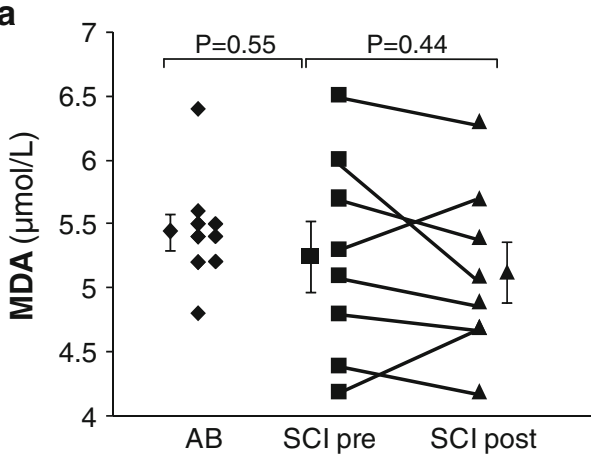

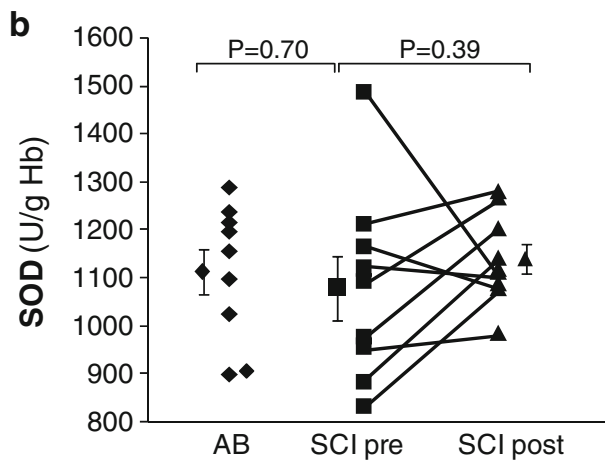

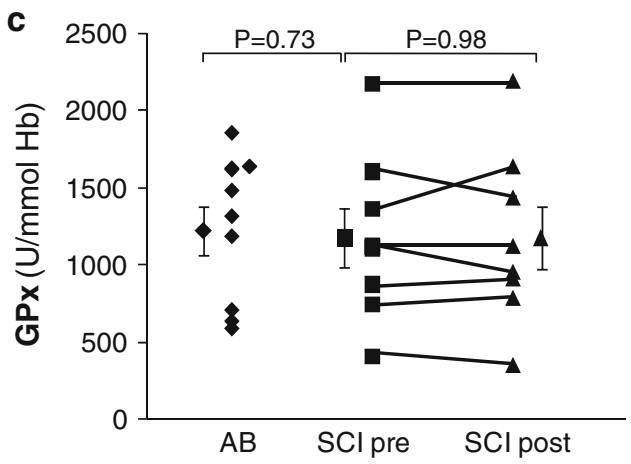


( $P=0.14,0.39$ and 0.98 , respectively). After 8 weeks of FES training, MDA, SOD and GPx levels were not different at baseline $(P=0.44,0.39$ and 0.98 , respectively; Fig. $1 \mathrm{a}-\mathrm{c})$ or post-exercise $(P=0.92,0.82$ and 0.37 , respectively; Fig. 3a-c) compared to pre-training.

\section{Discussion}

The purpose of this study was to gain more insight into the (anti)oxidative balance under basal conditions and in response to physical exercise in individuals with a chronic SCI as compared to ambulatory subjects. We found that healthy individuals having an SCI without cardiovascular or metabolic disease demonstrate comparable baseline levels of oxidative stress and antioxidative capacity as AB subjects, which suggests that subjects with a chronic SCI demonstrate a preserved (anti)oxidative balance. However, the relation between physical fitness level and oxidative stress in subjects with an SCI indicates that higher fitness levels in subjects with SCI are associated with a better oxidative status. Nonetheless, both a single bout of FES exercise and 8 weeks of FES exercise training had no effect on levels of oxidative stress and antioxidative capacity in subjects having a chronic SCI subjects. These findings suggest that despite the potentially beneficial vascular effects of FES exercise (de Groot et al. 2005; Hopman et al. 2002; Thijssen et al. 2006), training as applied in this study was insufficient to induce changes in the (anti)oxidative balance in subjects with an SCI.

In contrast to our hypothesis, we found preserved baseline levels of MDA, SOD and GPx in individuals with a chronic SCI. A reasonable explanation for this finding may relate to the activity level of our participants. In our study, we included sedentary as well as relatively active subjects with an SCI, who performed up to $6 \mathrm{~h}$ of sports a week (e.g. wheelchair basketball, rugby and hand biking). Interestingly, a moderate inverse correlation was found between maximal workload during the maximal hand bike test and baseline levels of oxidative stress. This indicates that a higher aerobic fitness level is associated with a beneficial lower oxidative stress level in subjects with SCI, a finding that was described previously in able-bodied subjects (Bloomer and Fisher-Wellman 2008). This correlation may, at least partly, explain the findings of a previous study which reported a strong correlation between the severity of a cervical lesion and (anti)oxidant status. They reported that a severe spinal cord lesion, which is associated with lower physical activity levels, is related to intensification of oxidative stress and a decrease in
Fig. 3 Responses of MDA malondialdehyde (a), SOD superoxide dismutase (b) and GPx glutathione peroxidase (c) levels (delta, \%) to one bout of exercise in individuals with spinal cord injury (SCI) pre and post 8 weeks FES exercise training. Data are presented as individual blood values and mean \pm SEM
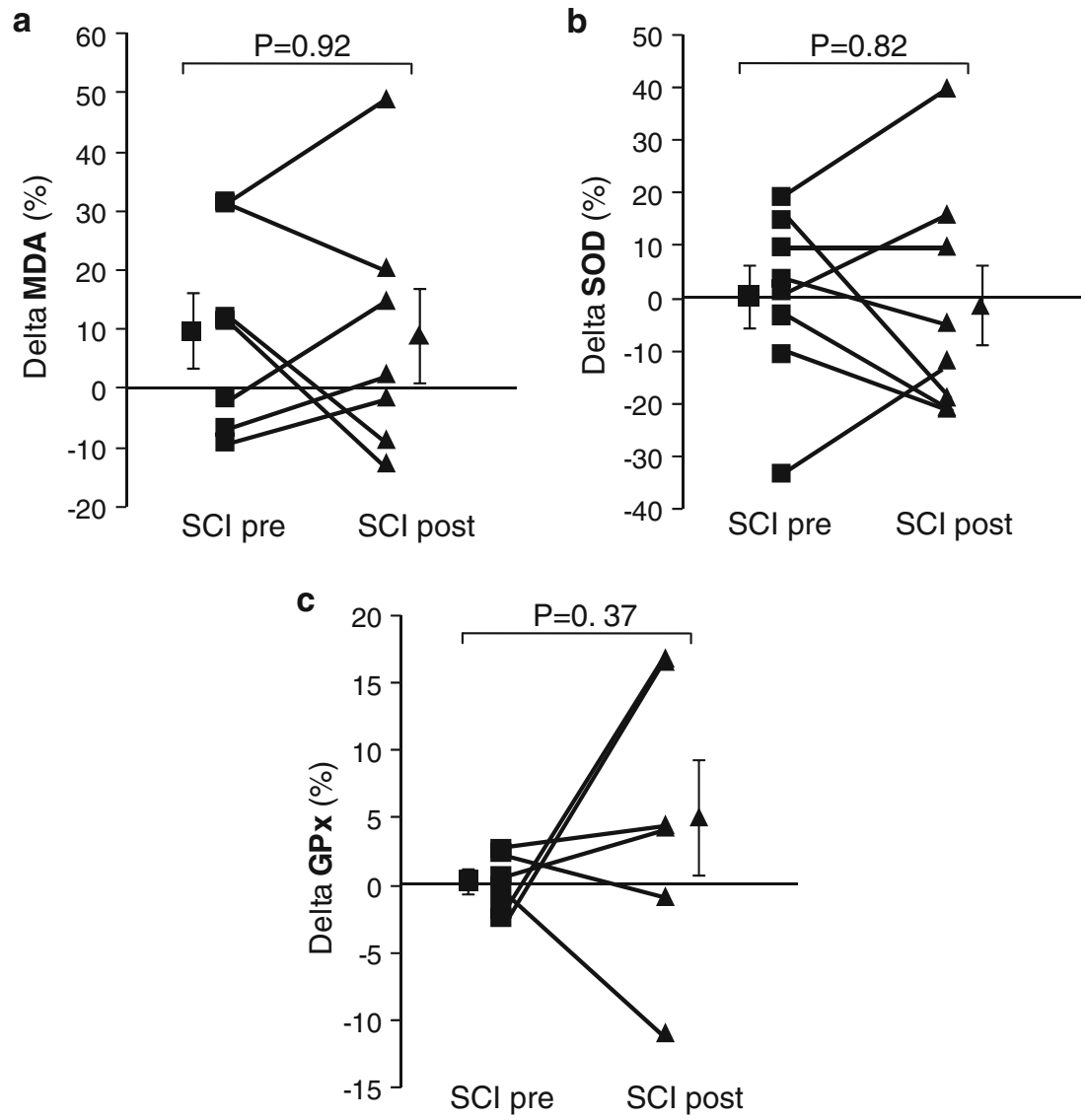
antioxidant potential (Wozniak et al. 2003). In line with our hypothesis, oxidative stress levels increase markedly during the first weeks of strict immobilization after the onset of an SCI (Liu et al. 1999). In parallel, also 2-3 months of strict bed rest in healthy subjects causes an increase in oxidative stress, while returning to initial a priori physical activity normalizes (anti)oxidative status (Margaritis et al. 2009). This may also explain why we found a preserved oxidative status in individuals with long-standing SCI, as their activity level in daily living will be increased when compared with strict bed rest.

Despite the moderate correlation between physical activity level and oxidative stress in SCI, levels of MDA, SOD and GPX were unaltered after one bout of FES exercise and 8 weeks of FES exercise training. These findings are in contrast with our hypothesis and several previous studies in rodents (Kanter et al. 1985; Kim et al. 1996; Laughlin et al. 1990), and healthy (Alessio 1993; Evelo et al. 1992; Franzoni et al. 2005; Robertson et al. 1991) and coronary artery diseased humans (Adams et al. 2005; Edwards et al. 2004; Leaf et al. 1999) demonstrating an improvement in (anti)oxidative status after short-term exercise or long-term training. Nonetheless, some studies have also reported unchanged levels of SOD and GPx after intermittent sprint cycle training in healthy subjects (Hellsten et al. 1996), while others found no changes in SOD in trained rats (Alessio and Goldfarb 1988; Laughlin et al. 1990) and in young men after running training (Ohno et al. 1988). It was hypothesized that exercise intensity level is crucial to induce adaptation processes and may explain the conflicting results of previous studies (Goto et al. 2007).

High-intensity exercise $\left(75-100 \% \mathrm{VO}_{2} \max \right)$ has been shown to result in increased oxidative stress (Goto et al. 2007; Wang et al. 2000) and elevated antioxidative enzyme activities (Powers et al. 1994). Accordingly, high-intensity exercise seems to be most appropriate to alter oxidative status. Based on a previous study, common FES exercise as applied in our study will elevate heart rate to $\sim 123 \mathrm{bpm}$ and increase oxygen uptake up to $63 \%$ of the $V \mathrm{O}_{2} \max$ (Holme et al. 2001). Accordingly, FES cycling exercise in individuals with an SCI does not represent high-intensity exercise and, therefore, may be insufficient to alter (anti)oxidative status. Indeed, even the last FES cycling exercise bout, which was performed at a markedly higher workload and exercise intensity than the first test, did not alter the oxidative stress level in subjects with SCI. A recent study showed that a stronger exercise stimulus can be induced by several technical modifications to the leg cycle ergometer (Janssen and Pringle 2008). One might, therefore, speculate that by extending the duration of the FES exercise training period or by technical modifications, FES exercise would be sufficient to induce changes in (anti)oxidative status. Furthermore, in contrast to FES exercise alone, voluntary arm cranking (alone or combined with FES cycling) may be more suitable to achieve higher exercise intensities. In future studies, measuring MDA levels after the physical fitness test is of additional value to determine if oxidative stress is indeed increased by maximal arm crank exercise.

Another reason that may explain that our findings are related to the muscles of the individuals with SCI. Up-regulation of antioxidative enzymes may be limited to highly oxidative skeletal muscles (Laughlin et al. 1990). Muscles of individuals with SCI show a shift in muscle fiber type towards type IIB (fast twitch, fast fatigable and glycolytic) fibers (Round et al. 1993), which might impair the ability to increase antioxidative enzymes by training in individuals with SCI.

\section{Clinical relevance}

Based on the inverse relation between aerobic fitness and level of oxidative stress in SCI individuals, we recommend individuals with a chronic SCI to increase their physical activity level to maintain or even improve their upper-body physical fitness to prevent high levels of oxidative stress. Although a direct relation between oxidative stress levels and cardiovascular risk in individuals with an SCI is unknown, we expect low oxidative stress levels and elevated levels of antioxidative enzymes to reduce cardiovascular risk in SCI.

\section{Limitations}

Local measurements of (anti)oxidative status in muscle tissue are preferred over blood analysis, as oxidative stress is primarily induced by the mitochondria. However, indirect (anti)oxidative status assessment from blood samples is commonly accepted and widely applied, while it also has important practical advantages (Edwards et al. 2004; Evelo et al. 1992; Leaf et al. 1999; Ohno et al. 1988). In addition, both SOD and GPx measurements are dependent on specific substrates (selenium and manganese, respectively), which are influenced by dietary intake. Participants registered detailed food intake 3 days prior to baseline measurements, and were asked to keep this similar before the pre- and post-training measurements. This procedure minimized the impact of diet for the comparison between pre- and post-training. We used maximal workload to represent physical fitness in individuals with SCI. This was done because, due to technical problems, we were not able to use oxygen uptake values measured during the physical fitness (arm crank) test. Finally, the relatively small sample size unlikely confounded our results, given the well-controlled, within-subject design 
and the relatively small variation in the data between- and within subjects.

In conclusion, our results indicate that healthy, nonmedicated subjects with a chronic SCI lesion have preserved baseline levels of oxidative stress and antioxidative capacity when compared with able-bodied subjects. However, within the group of subjects with an SCI, a higher aerobic fitness is associated with higher levels of oxidative stress demonstrating the importance to maintain or improve a high physical fitness. Antioxidant capacity is not compromised in moderately active individuals with an SCI. In addition, common FES exercise training does not result in acute or long-term changes in (anti)oxidative status in individuals with an SCI, which is most likely explained by the relatively low-intensity stimulus provided by FES exercise.

Acknowledgments We would like to acknowledge Lieke de Vries for her assistance during the training. NTLvD is supported by the Netherlands Heart Foundation (Grant NHS 2005B113).

\section{Conflict of interest statement None.}

Open Access This article is distributed under the terms of the Creative Commons Attribution Noncommercial License which permits any noncommercial use, distribution, and reproduction in any medium, provided the original author(s) and source are credited.

\section{References}

Adams V, Linke A, Krankel N, Erbs S, Gielen S, Mobius-Winkler S, Gummert JF, Mohr FW, Schuler G, Hambrecht R (2005) Impact of regular physical activity on the $\mathrm{NAD}(\mathrm{P}) \mathrm{H}$ oxidase and angiotensin receptor system in patients with coronary artery disease. Circulation 111:555-562

Alessio HM (1993) Exercise-induced oxidative stress. Med Sci Sports Exerc 25:218-224

Alessio HM, Goldfarb AH (1988) Lipid peroxidation and scavenger enzymes during exercise: adaptive response to training. J Appl Physiol 64:1333-1336

Bloomer RJ, Fisher-Wellman KH (2008) Blood oxidative stress biomarkers: influence of sex, exercise training status, and dietary intake. Gend Med 5:218-228

de Groot P, Crozier J, Rakobowchuk M, Hopman M, MacDonald M (2005) Electrical stimulation alters FMD and arterial compliance in extremely inactive legs. Med Sci Sports Exerc 37:1356-1364

Edwards DG, Schofield RS, Lennon SL, Pierce GL, Nichols WW, Braith RW (2004) Effect of exercise training on endothelial function in men with coronary artery disease. Am J Cardiol 93:617-620

Evelo CT, Palmen NG, Artur Y, Janssen GM (1992) Changes in blood glutathione concentrations, and in erythrocyte glutathione reductase and glutathione S-transferase activity after running training and after participation in contests. Eur J Appl Physiol Occup Physiol 64:354-358

Franzoni F, Ghiadoni L, Galetta F, Plantinga Y, Lubrano V, Huang Y, Salvetti G, Regoli F, Taddei S, Santoro G, Salvetti A (2005) Physical activity, plasma antioxidant capacity, and endothelium- dependent vasodilation in young and older men. Am J Hypertens 18:510-516

Goto C, Nishioka K, Umemura T, Jitsuiki D, Sakagutchi A, Kawamura M, Chayama K, Yoshizumi M, Higashi Y (2007) Acute moderate-intensity exercise induces vasodilation through an increase in nitric oxide bioavailiability in humans. Am J Hypertens 20:825-830

Hellsten Y, Apple FS, Sjodin B (1996) Effect of sprint cycle training on activities of antioxidant enzymes in human skeletal muscle. J Appl Physiol 81:1484-1487

Holme E, Mohr T, Kjaer M, Nielsen B (2001) Temperature responses to electrically induced cycling in spinal cord injured persons. Med Sci Sports Exerc 33:431-435

Hopman MT, Groothuis JT, Flendrie M, Gerrits KH, Houtman S (2002) Increased vascular resistance in paralyzed legs after spinal cord injury is reversible by training. J Appl Physiol 93:1966-1972

Janssen TW, Pringle DD (2008) Effects of modified electrical stimulation-induced leg cycle ergometer training for individuals with spinal cord injury. J Rehabil Res Dev 45:819-830

Ji LL (1999) Antioxidants and oxidative stress in exercise. Proc Soc Exp Biol Med 222:283-292

Kanter MM, Hamlin RL, Unverferth DV, Davis HW, Merola AJ (1985) Effect of exercise training on antioxidant enzymes and cardiotoxicity of doxorubicin. J Appl Physiol 59:1298-1303

Kim JD, McCarter RJ, Yu BP (1996) Influence of age, exercise, and dietary restriction on oxidative stress in rats. Aging (Milano) $8: 123-129$

Kocak H, Oner-Iyidogan Y, Gurdol F, Kocak T, Esin D (2005) The relation between serum MDA and cystatin $\mathrm{C}$ levels in chronic spinal cord injury patients. Clin Biochem 38:1034-1037

Laufs U, Wassmann S, Czech T, Munzel T, Eisenhauer M, Bohm M, Nickenig G (2005) Physical inactivity increases oxidative stress, endothelial dysfunction, and atherosclerosis. Arterioscler Thromb Vasc Biol 25:809-814

Laughlin MH, Simpson T, Sexton WL, Brown OR, Smith JK, Korthuis RJ (1990) Skeletal muscle oxidative capacity, antioxidant enzymes, and exercise training. J Appl Physiol 68:23372343

Laughton GE, Buchholz AC, Martin Ginis KA, Goy RE (2009) Lowering body mass index cutoffs better identifies obese persons with spinal cord injury. Spinal Cord 47(10):757-762

Leaf DA, Kleinman MT, Hamilton M, Deitrick RW (1999) The exercise-induced oxidative stress paradox: the effects of physical exercise training. Am J Med Sci 317:295-300

Liang H, Chen D, Wang Y, Rimmer JH, Braunschweig CL (2007) Different risk factor patterns for metabolic syndrome in men with spinal cord injury compared with able-bodied men despite similar prevalence rates. Arch Phys Med Rehabil 88:1198-1204

Liu D, Liu J, Wen J (1999) Elevation of hydrogen peroxide after spinal cord injury detected by using the Fenton reaction. Free Radic Biol Med 27:478-482

Margaritis I, Rousseau AS, Marini JF, Chopard A (2009) Does antioxidant system adaptive response alleviate related oxidative damage with long term bed rest? Clin Biochem 42:371-379

Myers J, Lee M, Kiratli J (2007) Cardiovascular disease in spinal cord injury: an overview of prevalence, risk, evaluation, and management. Am J Phys Med Rehabil 86:142-152

Ohno H, Yahata T, Sato Y, Yamamura K, Taniguchi N (1988) Physical training and fasting erythrocyte activities of free radical scavenging enzyme systems in sedentary men. Eur J Appl Physiol Occup Physiol 57:173-176

Powers SK, Jackson MJ (2008) Exercise-induced oxidative stress: cellular mechanisms and impact on muscle force production. Physiol Rev 88:1243-1276 
Powers SK, Criswell D, Lawler J, Ji LL, Martin D, Herb RA, Dudley $G$ (1994) Influence of exercise and fiber type on antioxidant enzyme activity in rat skeletal muscle. Am J Physiol 266:R375R380

Robertson JD, Maughan RJ, Duthie GG, Morrice PC (1991) Increased blood antioxidant systems of runners in response to training load. Clin Sci (Lond) 80:611-618

Round JM, Barr FM, Moffat B, Jones DA (1993) Fibre areas and histochemical fibre types in the quadriceps muscle of paraplegic subjects. J Neurol Sci 116:207-211

Thijssen DH, Ellenkamp R, Smits P, Hopman MT (2006) Rapid vascular adaptations to training and detraining in persons with spinal cord injury. Arch Phys Med Rehabil 87:474-481

van de Kerkhof J, Schalkwijk CG, Konings CJ, Cheriex EC, van der Sande FM, Scheffer PG, ter Wee PM, Leunissen KM, Kooman
JP (2004) Nepsilon-(carboxymethyl)lysine, nepsilon-(carboxyethyl)lysine and vascular cell adhesion molecule-1 (VCAM-1) in relation to peritoneal glucose prescription and residual renal function; a study in peritoneal dialysis patients. Nephrol Dial Transplant 19:910-916

Wang JS, Yang CF, Wong MK, Chow SE, Chen JK (2000) Effect of strenuous arm exercise on oxidized-LDL-potentiated platelet activation in individuals with spinal cord injury. Thromb Haemost 84:118-123

Wozniak A, Kasprzak HA, Wozniak B, Drewa G, Beuth W, Sniegocki M, Grzelak L (2003) Lipid peroxidation and antioxidant potential in patients with cervical spinal cord injury. Neurol Neurochir Pol 37:1025-1035 (discussion 1036)

Yorek MA (2003) The role of oxidative stress in diabetic vascular and neural disease. Free Radic Res 37:471-480 